\title{
Procesos tafonómicos en una encrinita regional pensilvánica (Atokano), Sonora, México
}

\author{
Catalina Gómez-Espinosa ${ }^{1}$ \& Blanca Estela Buitrón-Sánchez ${ }^{2}$ \\ 1. Unidad Académica de Ciencias de la Tierra, Universidad Autónoma de Guerrero; c_gomez@ciencias.unam.mx \\ 2. Departamento de Paleontología, Instituto de Geología, Universidad Nacional Autónoma de México; \\ blancab@unam.mx
}

Recibido 13-I-2017. Corregido 27-III-2017. Aceptado 02-V-2017.

\begin{abstract}
Taphonomic processes in a Pennsylvanian regional encrinite (Atokano), Sonora, Mexico. Crinoids have an extensive fossil record and were a major component in Paleozoic marine communities; encrinites are common crinoid accumulations where specimens are totally disarticulated from the Ordovician to the Jurassic. In Sonora, Mexico, the crinoid skeleton was altered during diagenesis and replaced by silica. The corrasion was high; the incrustation low and there is evidence of dissolution and compaction. This is a sedimentological type accumulation in a high energy environment of allochthonous organism transported in a debris flow, deposited below the storm wave base in an outer ramp. Our results can be correlated with regional encrinites prevalent in the Pennsylvanian cyclothems of North America. Rev. Biol. Trop. 65(Suppl. 1): S147-S159. Epub 2017 November 01.
\end{abstract}

Key words: Regional Encrinite; Taphonomical processes; Sedimentological concentration; Pennsylvanian; Carboniferous.

Los equinodermos tienen un amplio registro fósil y una extensa distribución ambiental, este phylum se diversificó durante el Paleozoico temprano y desarrolló una amplia plasticidad morfológica (Kroh \& Nebelsick, 2010); el estudio del tipo de preservación de estos organismos es muy útil para su aplicación en estudios paleobiológicos (Nebelsick, 2004). Entre los equinodermos destacan los crinoideos como un componente principal de las comunidades marinas del Paleozoico (Sepkoski, 1981). Algunos autores han propuesto que los equinodermos presentan una ventaja inherente para los estudios de tipo tafonómico debido a que su endoesqueleto compuesto por calcita alta en magnesio, muestra un gran potencial de fosilización, además el endoendoesqueleto de los crinoideos está compuesto por múltiples numerosas placas en la columna, el cáliz y los brazos (Kroh \& Nebelsick, 2010), mientras que los ejemplares completos son muy útiles para interpretar patrones ecológicos, los fragmentos son más sensitivos a los procesos tafonómicos (Perry, 1996; Brachert, Braga, \& Martin, 1998). Aunque un endoesqueleto formado por múltiples elementos implica algunas limitaciones como la estimación del número de ejemplares (Gilinsky \& Bennington, 1994).

Los equinodermos han sido ampliamente utilizados en estudios tafonómicos desde el punto de vista descriptivo (Donovan \& Gordon, 1993; Sumrall, 2001; Donovan \& Clements, 2002; Cornell, Brett, \& Sumrall, 2003; Twichett et al., 2005; Wetzel \& Meyer, 2006); de la comparación del potencial de preservación (Meyer, Ausich, \& Terry, 1989); de los patrones tafonómicos y los análisis de tafofacies (Nebelsick, 1999 a, b) y de la caracterización de gradientes ambientales (Ausich \& Sevastopulo, 1994; Kroh \& Nebelsick, 2003; 
Gahn \& Baumiller, 2004). Los estudios tafonómicos también se han utilizado como una herramienta para reconstruir la morfología funcional (Baumiller \& Hagdorn, 1995), para reconocer si la preservación de un tipo en particular refleja las limitantes paleoambientales (Dornbos \& Botjer, 2001) y para comparar entre posibles patologías o solo el resultado de procesos tafonómicos (Schneider, 1989).

En el estudio de la tafonomía actualística, se han realizado experimentos para entender el tipo de descomposición y tiempo de desarticulación entre los diferentes grupos de equinodermos (Kidwell \& Baumiller, 1990; Greenstein, 1991; Ausich \& Baumiller, 1993; Baumiller Llewellyn, Messing, \& Ausich. 1995); mientras que otros autores se han interesado exclusivamente en la investigación de los procesos de fragmentación para utilizarlos en la comparación con el registro fósil (Kidwell \& Baumiller, 1990; Greenstein, 1991; Gordon \& Donovan, 1992; Ausich \& Baumiller, 1993; Lewellyn \& Messing, 1993; Baumiller et al., 1995; Nebelsick, 1999b).

Los conjuntos de crinoideos paleozoicos muestran dos distintos tipos de preservación, los crinoideos articulados son considerados como depósitos tipo Lagersttäten y las acumulaciones de placas columnales desarticuladas forman encrinitas.

Los estudios tafonómicos en crinoideos se han basado principalmente en los ejemplares bien preservados (Donovan, 1996) resaltando los depósitos de tipo lagerstätten (e. g. Baumiller \& Gahn, 2004; Meyer \& Milsom, 2001). Sin embargo, los crinoideos muy raramente se preservan como ejemplares completos, debido a que se desarticulan muy rápidamente después de la muerte de los individuos y quedan mayormente representados como partes del cáliz, braquiolas, placas columnales aisladas (Buitrón et al., 2007; Thomka, Mosher, Lewis, \& Pabian, 2012).

La mayoría de los estudios de crinoideos desarticulados del Paleozoicos han sido con fines descriptivos para proponer algunas clasificaciones parataxonómicas de los morfotipos de las placas (Thomka et al., 2012). Los restos de elementos de endoesqueletos de crinoideos fueron muy comunes en sedimentos del Paleozoico y algunas veces tan abundantes y diversos que formaron capas de rocas llamadas encrinitas. Una encrinita se ha definido como grainstone y packstone formados por más del $50 \%$ de su volumen por detritos de pelmatozoarios (Wright, 1934). Las encrinitas son comunes en sedimentos del Ordovícico al Jurásico, en Norte América el mayor desarrollo de éstas fue durante el Misisípico temprano (Osageano) (Ausich, 1997; Ginsburg, 2005). El análisis de tipo tafonómico de los elementos desarticulados de los crinoideos, especialmente en las encrinitas paleozoicas, ha sido escasamente estudiado por Thomka et al. (2011). Entre las investigaciones enfocadas a encrinitas paleozoicas y mesozoicas se encuentran las de Holterhoff (1996), Phelps \& Droser (2003), Ginsburg (2005), Hunter \& Zonnevel (2008), Hunter \& Clark (2009), Hunter \& Underwood (2009) y Thomka et al. (2012). En estos estudios se demostró que las encrinitas pueden ser utilizadas como herramienta para la recostrucción de los paleoambientes en que habitaban los crinoideos.

Las encrinitas representan una litofacies extinta (Hunter \& Zonneveld, 2008) debido a que actualmente no se están formando sedimentos donde los contribuidores predominantes sean los crinoideos y por tanto, se carece de analogías directas para el estudio de los depósitos y procesos de los sedimentos antiguos (Meyer \& Meyer, 1986). El objetivo de esta investigación es la caracterización de los procesos bioestratinómicos y fosildiagenéticos de una encrinita Pensilvánica de Sonora, México.

\section{MATERIALES Y MÉTODOS}

Locálización: La Sierra Agua Verde se encuentra localizada a 120 kilómetros al este de la ciudad de Hermosillo, en el Estado de Sonora. Abarca una superficie de aproximadamente $255 \mathrm{~km}^{2}$ y se encuentra entre los paralelos $109^{\circ} 56^{\prime} 24^{\circ}-109^{\circ} 47^{\prime} 09^{\circ} \mathrm{W}$ y $29^{\circ} 17^{\prime} 45^{\circ}-29^{\circ} 08^{\prime} 37^{\circ} \mathrm{N}$ (Fig. 1). Las rocas que afloran en esta Sierra tienen una edad que 


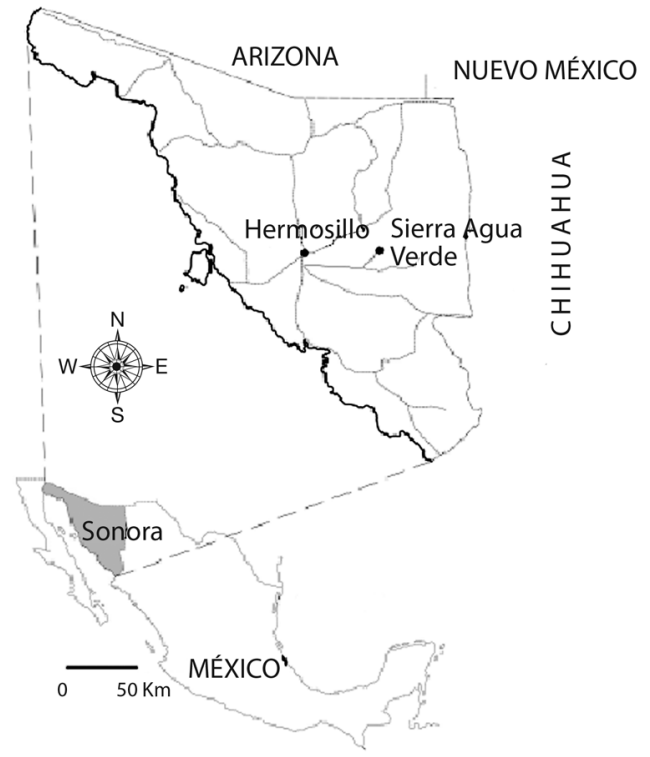

Fig. 1. Localización de la Sierra Agua Verde.

Fig. 1. Localitation of the Sierra Agua Verde.

comprende del Neoproterozoico (?) al Pérmico (Ochoa-Granillo \& Sosa-León, 1993).

El estudio se realizó a lo largo de una secuencia de 100 metros de potencia en un afloramiento de la formación La Joya locálizado en el "Rancho Agua Caliente", esta secuencia fue muestreada capa por capa para identificar los estratos que correspondían a las encrinitas (Fig. 2). Se recolectaron quince muestras aleatorias en los tres niveles marcados como "crinoideos" de la figura 2. Las muestras fueron procesadas de acuerdo al método propuesto por Thomka et al. (2012) para la separación de la matriz de los restos fósiles y la recuperación de los ejemplares. Cada muestra contenía en promedio unos 100 ejemplares de fragmentos de placas columnares. El análisis tafonómico se realizó utilizando una combinación de los métodos propuesto por Ausich \& Baumiller (1993), Baumiller \& Ausich (1992), Holterhoff (1996) y Thomka et al. (2012). Los fósiles de crinoideos fueron estudiados usando un microscopio binocular. Las características bioestratinómicas consideradas dentro del análisis fueron el grado de articulación, fragmentación, corrasión (término que se utiliza

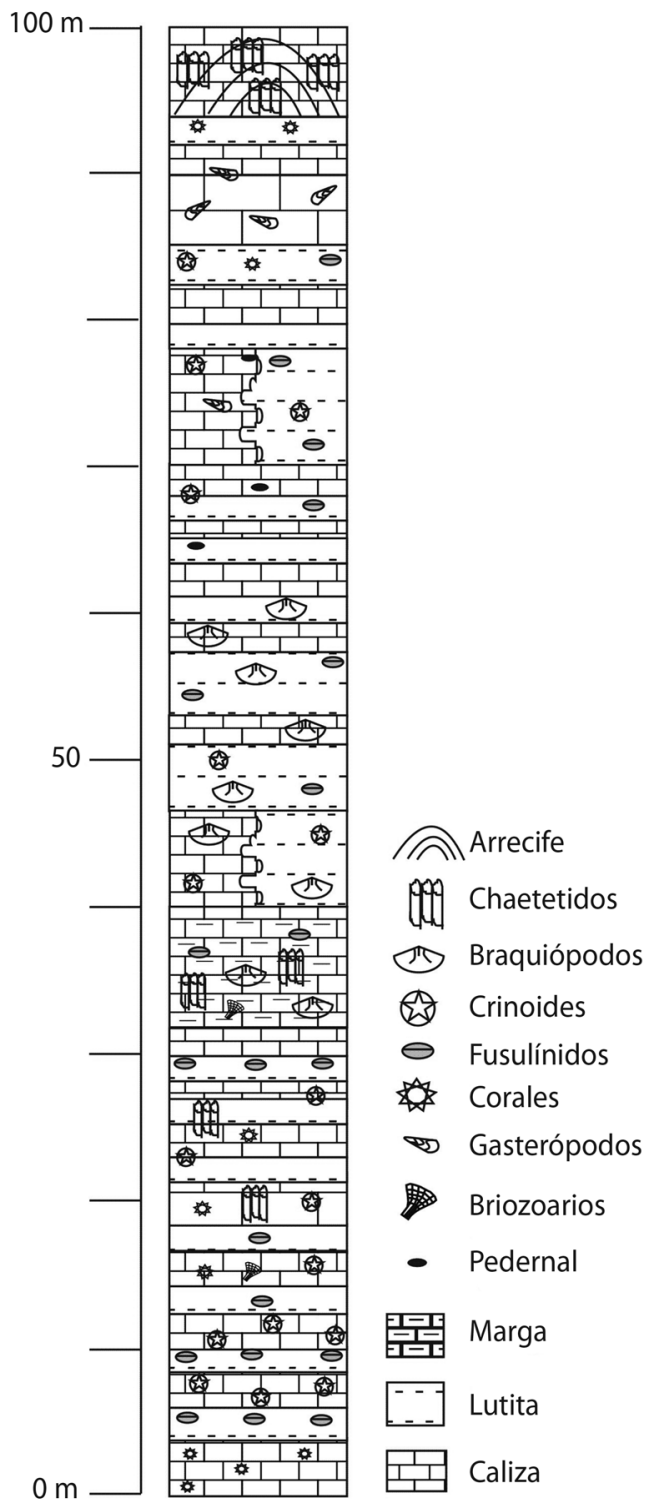

Fig. 2. Columna estratigráfica de la formación La Joya en la Sierra Agua Verde.

Fig. 2. Stratigraphic column of La Joya Formation in Sierra Agua Verde.

para englobar los procesos de abrasión mecánica, corrosión química y erosión biológica), incrustación y orientación. Las características fosildiagenéticas estudiadas fueron la compactación y la disolución. Las escalas utilizadas para el grado de articulación fueron: grado 1 ejemplar completamente articulado, grado 2 
cáliz articulado, grado 3 ejemplar desarticulado en fragmentos de placas columnares y grado 4 ejemplares desarticulados en placas aisladas. Para el grado de incrustación se utilizó la escala propuesta por Brett \& Bordeaux (1990) y para el grado de corrasión se aplicó la escala usada por Llewellyn \& Messing (1993). La fragmentación y la disolución diagenética fueron clasificadas únicamente bajo los criterios de presente o ausente.

La compactación fue estudiada como una característica auxiliar para identificar la orientación relativa de los ejemplares dentro del sustrato, en tanto que la orientación se consideró de acuerdo a la posición de los ejemplares de acuerdo a la base y cima de las capas que los contenían considerando la posición paralela, cuasivertical o vertical. Los datos sobre la orientación se tomaron antes de la separación del material de la matriz donde estaban contenidos y solamente se hizo para los ejemplares que estaban visibles dentro de las muestras de roca. La escala utilizada para la cuantificación de la compactación fue: grado 1 sin compactación, grado 2 ligera compactación lateral, grado 3 bordes compactados, grado 4 alta compactación lateral que ocasiona la deformación y elongamiento del ejemplar.

La formación La Joya corresponde a una unidad informal del Pensilvánico (Atokano) (Gómez-Espinosa et al., 2008), aparece como un sistema transgresivo (TST), donde la litología predominante son cálizas fosilíferas marinas intercaladas con limolitas y margas con nódulos de pedernal (Fig. 2). Los crinoideos están representados por once especies identificadas con base en la parataxonomía propuesta por Moore \& Jeffords (1968), y corresponden a Cyclocaudex insaturatus Moore y Jeffords, (1968), Cyclocrista martini Miller, Heterosteleschus keithi Miller, Lamprosterigma erathense Moore y Jeffords, (1968), L. mirificum Moore y Jeffords, (1968), Mooreanteris waylandensis Miller, Pentagonopterix insculptus Moore y Jeffords, (1968), Preptopremnum laeve Moore y Jeffords, (1968), P. rugosum Moore y Jeffords, (1968), Cycloscapus laevis Moore y Jeffords, (1968) y Pentaridica simplicis Moore y Jeffords, (1968) (Buitron et al., 2007). El conjunto fósil también contiene algas calcáreas, foraminíferos, esponjas, briozoarios, corales solitarios, braquiópodos y gasterópodos.

\section{RESULTADOS}

Se recolectó un total de 1710 ejemplares, todos ellos corresponden a crinoideos desarticulados, representados por fragmentos de columnas $(91 \%)$ y solo escasas placas desarticuladas aisladas (9\%), ésta característica representa un grado de articulación bajo y muy bajo respectivamente. La principal causa de alteración de los restos esqueltales fue la corrasión (Fig. 3B), el 44.6\% de los ejemplares muestran los bordes y el crenularium removidas (grado tafonómico 5) (Fig. 4.A4), mientras que el $26.8 \%$ muestra los bordes redondeados y el crenularium muy erosionado (grado tafonómico 4) (Fig. 4.A3), el 18.8\% de los ejemplares presentan los bordes erosionados y el crenularium reducido (grado tafonómico 3) (Fig. 4.A2) y solo $9.8 \%$ de los ejemplares presentan el crenularium con una ligera abrasión (grado tafonómico 2) (Fig. 4.A1). La característica de fragmentación tiene una representación muy baja en las muestras pues solo un $8 \%$ de los ejemplares la presentan (Fig. $3 \mathrm{C})$. Los restos de crinoideos se depositaron sin una orientación preferente, el $46 \%$ de los ejemplares estaban paralelos al sustrato, 14\% de forma cuasivertical y $40 \%$ orientados de manera paralela al estrato (Fig. 3D). La incrustación se presenta en $12.3 \%$ de la muestra, los grados de incrustación son 1, 2, 3 y 5 . La mayor parte de los ejemplares $(87.7 \%)$ tienen un grado de incrustación 1 que corresponde a la ausencia de incrustantes (Fig. 3E). Los incrustantes están representados por briozoarios tanto fenestélidos como rombopóridos (Fig. 4.B56) y por corales coloniales (Fig. 4.B1-4). La identificación taxonómica solo fue posible en algunos de los casos, el género Fenestella sp. se encontró como incrustante sobre las placas columnares del crinoideo Cyclocrista martini (Fig. 4.B7) y briozoarios del tipo rombopóridos se identificaron sobre placas columnares 


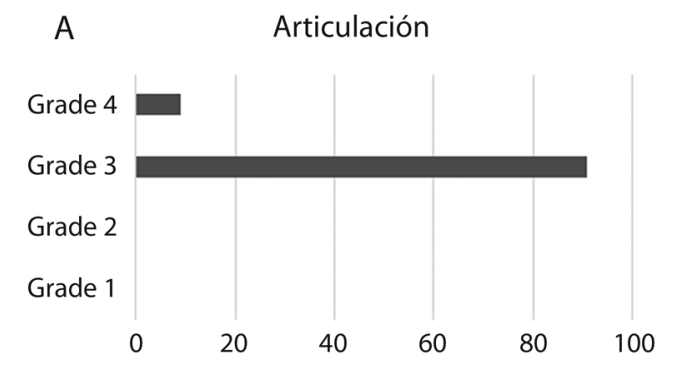

B Corrasión

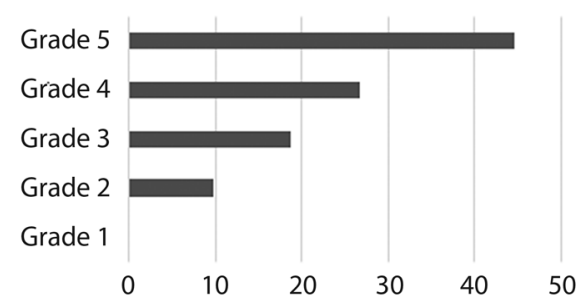

C

Fragmentación

D

Orientación

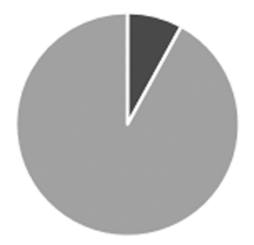

- Fragmentado " No fragmentado
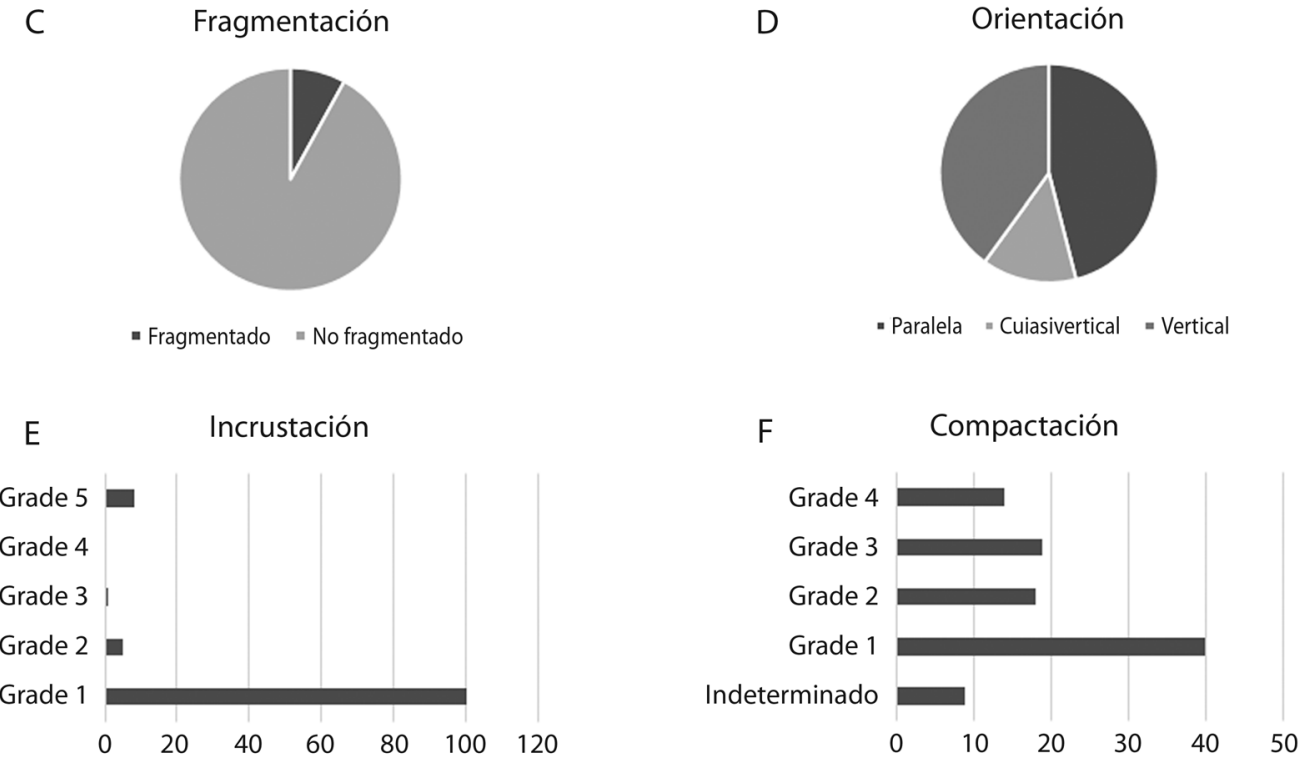

Fig. 3. Características bioestratinómicas analizadas. A: grado de articulación; B: grado de corrasión; C: grado de fragmentación; D: grado de orientación de los crinoideos con respecto a la base del estrato; E: grado de incrustación; F: grado de compactación.

Fig. 3. Biostratinomic characteristics analyzed. A: degree of articulation; B: degree of corrosion; C: degree of fragmentation; D: degree of orientation of the crinoids with respect to the base of the stratum; E: degree of inlay; F: degree of compaction.

de las especies Lamprosterigma mirificum (Fig. 4.A2), y Heterostelechus keithi (Fig. 4.A1); en tanto que los corales coloniales se identificaron creciendo sobre fragmentos de la columna de Lamprosterigma sp. (Fig. 4.A4).

Los depósitos de crinoideos estudiados corresponden a una textura tipo rudstone en una matriz de terrígenos y con ausencia de bioturbación. En sección delgada es evidente la presencia de microestructuras de oscículos de crinoideos preservadas por sustitución de cuarzo microcristalino. Respecto a la fosildiagénesis, el $100 \%$ de la muestra presenta disolución, sin embargo, es el resultado de diagénesis secundaria. En el caso de la compactación, ésta no se presenta en el $40.3 \%$ de los ejemplares (Fig. $3 \mathrm{~F}$ ), es evidente en el $50.9 \%$ de la muestra y no es posible cuantificarla en un $8.8 \%$ debido a que los ejemplares presentan un alto grado de disolución secundaria, que enmascara la presencia de la compactación. Del 50.9\% de los ejemplares que presentan compactación, un $18 \%$ corresponde a un grado bajo (Fig. 4.C1), un $19 \%$ a un grado medio (Fig. 4.C2) y un $14 \%$ 

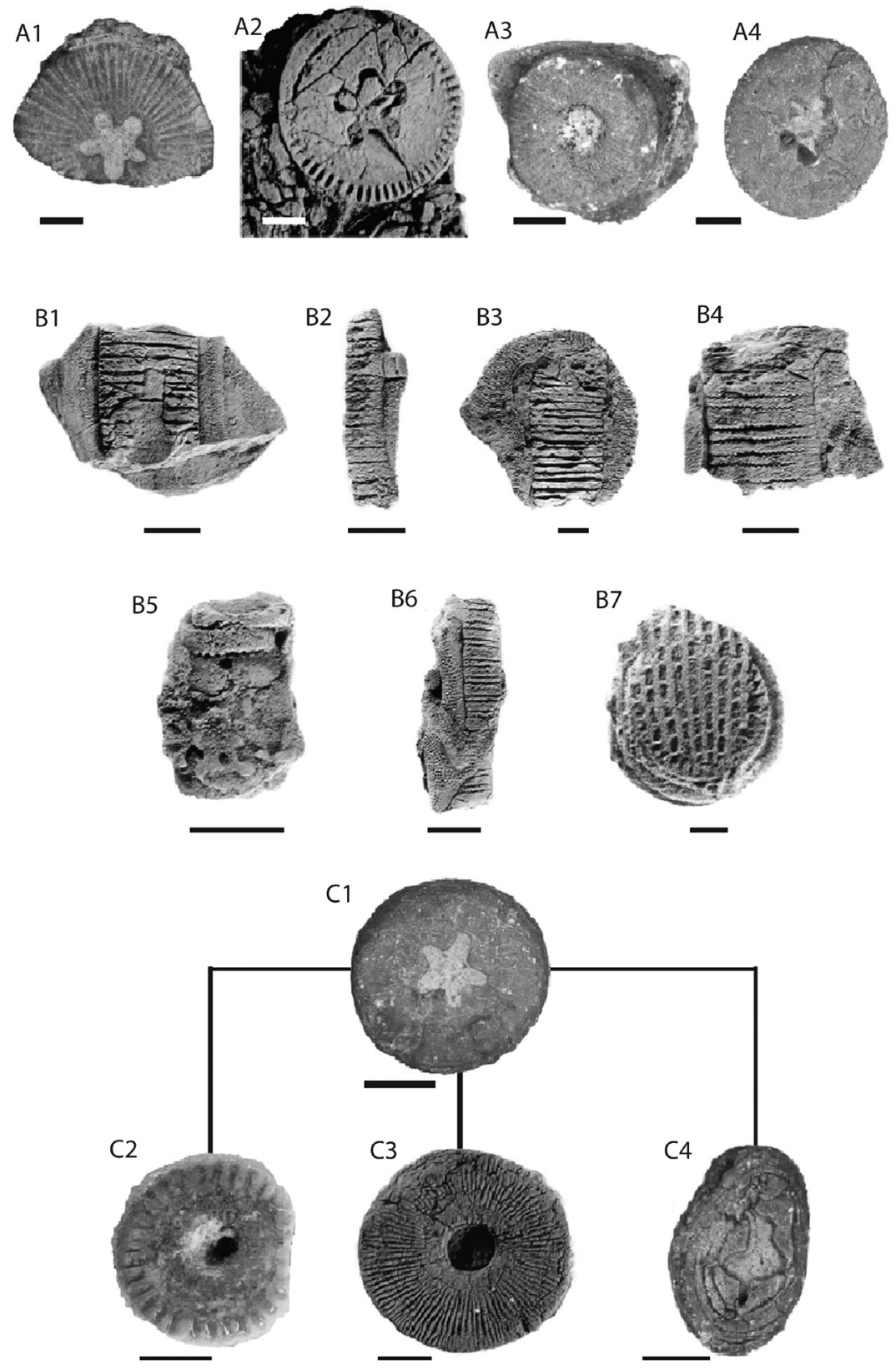

Fig. 4. Índice de corrasión en crinoideos. A1: abrasión menor de crenelas y bordes, A2: crenulación reducida y abrasión en los bordes, A3: crenulación casi indistinguible, bordes extensamente redondeados, A4: crenulación removida bordes ampliamente redondeados. Epibiontes sobre crinoideos. B1-B4: corales coloniales, B5-B6: briozoarios rombopóridos, B7: briozoarios fenestélidos. C: Grado de compactación en crinoideos: C1 sin compactación, C2 compactación lateral ligera, C3 bordes comprimidos, $\mathrm{C} 4$ placa deformada en forma ovoidal (Escala $5 \mathrm{~mm}$ ).

Fig. 4. Corrosion grades in crinoids. A1: lower abrasion of crenels and edges, A2: reduced crenulation and abrasion at edges, A3: almost indistinguishable crenulation, extensively rounded edges, A4: crenulation removed widely rounded edges. Epibionts on crinoids. B1-B4: colonial corals, B5-B6: romboporid bryozoans, B7: fenestellid bryozoans. C: Degree of compaction in crinoids: C1without compaction, C2 slight lateral compaction, C3 edges compressed, C4 plate deformed in ovoid form (5 mm scale). 
a un grado alto, estos últimos incluso muestran deformación en las placas las cuales se ven elongadas (Fig. 4.C3).

Caracterización de la tafofacies: La fauna dominante está representada por crinoideos epibentónicos que corresponden a once paraespecies. La fauna asociada está representada por taxa que poseen endoesqueletos de carbonato de calcio bajo en magnesio como los fusulínidos (foraminíferos bentónicos), briozoarios fenestélidos y rombopóridos con endoesqueletos arborescentes y braquiópodos espiriféridos y prodúctidos. Litológicamente corresponde a una marga con textura tipo rudstone. Tafonómicamte está caracterizado por crinoideos desarticulados en forma de fragmentos de columnas y una baja proporción de placas columnares aisladas, los grados de corrasión y compactación son de medios a altos. La orientación es aleatoria y el grado de incrustación y de fragmentación son bajos. Hay un evidente retrabajo, clasificación y transporte dentro de las litofacies.

\section{DISCUSIÓN}

Las encrinitas son un ejemplo de retroalimentación tafonómica (Kidwell \& Jablonski, 1983) por la predominancia de un tipo particular de grano esqueletal. Las características tafonómicas de los crinoideos están controladas tanto por factores intrínsecos (e.g. composición morfológica) como por factores extrínsecos (principalmente las condiciones paleoambientales). El grado de desarticulación está relacionado con ambos tipos de factores. En condiciones marinas normales, los equinodermos comúnmente se desarticulan en placas columnares en un periodo que va de 1 a 2 semanas, dependiendo del tipo de composición del endoesqueleto y de los factores ambientales. El análogo más cercano a los crinoideos paleozoicos (sésiles bentónicos), corresponde a los crinoideos actuales (plactónicos móviles), en el caso de los pelmatozoarios actuales los brazos y los cirros empiezan a desarticularse dentro de los primeros tres días después de su muerte, una vez transcurridos seis días tanto el cáliz como algunos de los segmentos de los brazos están desarticulados (Dornbos \& Bottjer, 2001). Se ha observado que la desarticulación de los crinoideos no presenta un patrón aleatorio, sino que es resultado de la descomposición posmorten de los diferentes tipos de tejidos de los que están formados (Baumiller \& Hagdorn, 1995). La articulación entre las columnas de las placas columnares de los crinoideos está formada por ligamentos y hay zonas (nodos) donde las columnas son estructuralmente más débiles. Cuando una columna empieza a desarticularse se rompe primero en los nodos, produciendo fragmentos columnares de una longitud muy similar. Finalmente, cuando todos los ligamentos se han deteriorado las columnas empiezan a desarticularse en placas individuales (Baumiller \& Ausich, 1992).

La encrinita en la formación La Joya está conformada por fragmentos de columnas y en un porcentaje muy bajo por placas columnales individuales, pero no se preservaron restos ni del cáliz ni de los brazos. Desafortunadamente, debido a la ausencia del cáliz, estos fósiles no pueden asignarse a un clado en particular y, por tanto, el grado de resistencia a la desarticulación no puede ser interpretada aplicando los estudios de tafonomía actualística. Sin embargo, puede interpretarse que la desarticulación debió empezar en el cáliz y los brazos y por eso no se preservaron. Posteriormente, se inició la desarticulación en los nodos en la zona de los ligamentos intercolumnares y los restos debieron quedar cubiertos por sedimentos. La ausencia del cáliz también puede ser interpretada como el resultado de una remoción selectiva por parte de los depredadores y no sólo debido a efectos tafonómicos (Meyer \& Meyer, 1986). La desarticulación en columnas de los tallos distales también puede haber sido resultado del ciclo normal de crecimiento, como respuesta a algún tipo de estrés ecológico o alguna catástrofe como una avalancha de sedimentos (Baumiller et al., 1995). Algunos autores incluso han llegado a sugerir que los crinoideos paleozoicos tenían la habilidad de sobrevivir aún después de la pérdida del cáliz 
y que esa es la razón por la cual las encrinitas están dominadas por fragmentos de columnas y columnas individuales (Donovan \& Schmith, 2001; Oji \& Amemiya, 1998). Respecto a la orientación de los restos de los crinoideos, la interpretación se dificulta debido a que las partículas cilíndricas tienen patrones muy complejos que no permiten una relación directa entre el tipo de transporte y la orientación (Schwarzacher, 1963). Debido a la composición y morfología del endoendoesqueleto de los crinoideos, este es muy resistente cuando están vivos, sin embargo, una vez muertos los restos son fácilmente corrosionados y transportados (Kroh \& Nebelsick, 2010). La corrasión es el factor que más altera la superficie de los crinoideos. La abrasión tiene una interpretación ambigua, pues puede ser el resultado del transporte de las partículas esqueletales in situ, causada por olas o corrientes en ambientes de alta energía, donde la abrasión es alta (Nebelsick, 1999b). La corrasion también puede interpretarse como evidencia de un transporte material hacia abajo en una zona de escarpe, y el transporte en este tipo de topografía puede haber aumentado el daño durante el proceso (Meyer \& Meyer, 1986).

Respecto a la interpretación de los epibiontes, en la mayoría de los casos, se considera que no puede demostrarse la interacción entre estos y los crinoideos del registro fósil, pues la asociación pudo ser en vida, como sustrato muerto o incluso como parásitos (Gluchowski, 2005). Los epibiontes estudiados en este caso, no tienen hábitos de parasitismo y no hay evidencia de deformación o de inhibición en las funciones vitales del crinoideo o que hayan sido causadas por los organismos que se encontraban viviendo sobre ellos. Las asociaciones en vida entre los crinoideos y sus epibiontes generalmente representan una interacción de tipo comensalista $\mathrm{u}$ oportunista por parte del epibionte; mientras que en las asociaciones de muerte, se utilizan exclusivamente como sustratos duros. En el caso particular de la asociación entre Fenestella sp. con Cyclocrista martini, la colonización por parte del briozoario se interpreta que ocurrió después de la muerte y desarticulación del crinoideo. En tanto, que la asociación entre los briozoarios rombopóridos y Lamprosterigma mirificum debió darse en vida, pues el briozoario, con una excelente preservación, creció alrededor de la columna.

La presencia de un sobrecrecimiento en las columnas de los crinoideos es una respuesta natural de los crinoideos recientes, este es el caso que se interpreta para la pluricolumna que tiene como epibionte a un coral colonial (Fig. 4. B2). Se sugiere una asociación en vida también entre el crinoideo y el coral colonial, esto se infiere debido a que hay un espacio lleno por matriz siliciclástica entre la columna silicificada del crinoideo y la base del crecimiento del coral, lo cual se interpreta como un sobrecrecimiento defensivo por parte del crinoideo. En el resto de los casos de incrustación, no es posible saber si la asociación se dio cuando los crinoideos estaban vivos o ya eran parte de las partículas sedimentarias. El bajo grado de incrustación también evidencía el depósito de los sedimentos en un ambiente caracterizado por un alto grado de turbidez, inestabilidad y condiciones anoxigénicas. El grado de fragmentación en este caso particular no se utilizó para realizar ningún tipo de interpretación debido a que, como resultado de la diagénesis secundaria se presenta una alta disolución la cual oscurece el porcentaje real de fragmentación.

El endoesqueleto de los crinoideos está compuesto por calcita alta en $\mathrm{Mg}$, este mineral es muy inestable y durante la diagénesis marina el primer paso implica la transformación a calcita baja en Mg (Nebelsick, 2004). La cementación también se inicia casi de inmediato después del decaimiento y descomposición del tejido (Kroh \& Nebelsick, 2010) y el reemplazamiento de los restos de crinoideos por sílice, indica un proceso diagenético temprano en sedimentos sobresaturados de sílice disuelto. La microestrucutra del endoesqueleto de los crinoideos es indicadora de un reemplazo directo de la calcita por sílice. 
Ambiente de depósito e implicaciones paleoambientales: Los equinodermos actuales habitan en aguas templadas principalmente en ambientes no tropicales (Nelson, 1988). Los restos desarticulados de los crinoideos se depositan y quedan sepultados cerca de la zona en donde se encontraban viviendo (Ausich Brett \& Hess, 1999) y los patrones de distribución se explican más que nada debido a la topografía local y a los factores hidrodinámicos (Llewellyn \& Messing, 1993).

Las comunidades de crinoideos recientes habitan en zonas con topografías con crestas y valles (Llewellyn \& Messing, 1993; Meyer \& Meyer, 1986) y cuando mueren sus restos son transportados hacia abajo del escarpe ya sea a través de un proceso activo o por gravedad.

Se han sugerido varios ambientes donde pudieron formarse las encrinitas, dentro de estos se ha sugerido que puedieron haberse formado en bancos de arena en aguas agitadas (Wilson, 1975), en ambientes por debajo de la base de la ola en condiciones normales en aguas profundas (Ausich, 1997) o en ambientes de plataformas relativamente someras (Goebel, 1991; Phelps \& Droser, 2003).

El movimiento hacia abajo en zonas escarpadas, puede explicar el alto grado de corrasión que muestran los ejemplares estudiados, aunque puede deberse también a un retrabajo in situ, a movimientos laterales a pequeña escala o incluso a una alta exposición de los restos en la interfase agua sedimento. El tiempo requerido de exposición o de transporte de los restos de crinoideos y su relación con los diferentes grados de abrasión no se conoce (Llewellyn \& Messing, 1993). Los restos de crinoideos que son transportados por flujo de sedimentos pueden tener una orientación horizontal, inclinada o vertical (Donovan \& Pickerill, 1995). El trasporte a bajas velocidades resulta en una orientación horizontal con respecto al plano de estratificación, cuando la velocidad del agua aumenta hay una probabilidad más alta de transporte caótico y por tanto una orientación aleatoria de los restos (Nebelsick \& Kroh, 2002). La orientación aleatoria de los crinoideos en la formación La Joya indica que los restos fueron transportados por flujo de sedimentos (Donovan \& Pickerill, 1995); los restos crinoidales pueden haber actuado también como un sustrato con movimientos episódicos (Phelps \& Droser, 2003). Este tipo de depósito es típico de la acción de tormentas (Parsons, Brett, \& Miller, 1988).

La acumulación de los restos originada por tormentas explica la ausencia de bioturbación dentro del estrato, la mala clasificación de los componentes fósiles y la orientación aleatoria de los crinoideos. La ausencia de bioturbación puede interpretarse como resultado de un depósito en un fondo marino pobremente oxigenado, o de acuerdo a Ginsburg (2005) como resultado de un sustrato inestable, que puede ser fácilmente retrabajado por el agua incluso con velocidades relativamente lentas. Las cálizas crinoidales son generalmente catalogadas como resultado de los flujos de gravedad (Flügel, 2004), pero más frecuentemente de tempestitas. Esta interpretación es aplicable a los depósitos de la Sierra Agua Verde. La acumulación de crinoideos estudiada corresponde al tipo de acumulación sedimentológica de acuerdo a la clasificación propuesta por Kidwell, Fürsich, \& Aigner, (1986), resultado de un proceso de acumulación hidraúlica de elementos autóctonos. Los depósitos de encrinita generalmente ocurren en entornos de plataformas relativamente someras (Goebel, 1991; Phelps \& Droser, 2003). De acuerdo con el modelo clásico de Burchette \& Wright (1992), la encrinita regional de la formación La Joya tuvo que haberse depositado en un ambiente de rampa externa por debajo de la base de la ola, pero con frecuente retrabajo por tormentas.

La acumulación de un gran número de restos de crinoideos en algunos estratos de la Sierra Agua Verde, indican condiciones óptimas para su desarrollo. Los parámetros que requieren los crinoideos para colonizar un área son una combinación de ausencia de turbulencia (Ausich, 1980), aguas bien oxigenadas, salinidad de entre 24 a 36 ppm y una alta disponibilidad de microplancton (Boolootian, 1966).

El establecimiento de las colonias de crinoideos requiere un sutrato sin disturbios 
físicos, estas condiciones predominan en el fondo marino por debajo de la zona infralitoral. Carozzi y Soderman (1962) sugirieron que las larvas de los crinoideos solo pueden establecerse en ambientes protegidos como depresiones topográficas. El aporte de material siliciclástico también es un factor importante para el desarrollo de amplias comunidades de crinoideos debido a que representan un recurso de nutrientes para organismos filtradores, el suministro de material siliciclástico puede ser el resultado de la sedimentación por tormentas o de la presencia de un recurso deltáico distal (Clayer, 2012). En el caso particular de las encrinitas estudiadas, no se puede inferir cuál fue el factor para el desarrollo de estas grandes comunidades de crinoideos, debido a que no se conoce la fuente de procedencia de las partículas siliciclásticas del afloramiento.

Basándose en las características bioestratinómicas y fosildiagenéticas, las encrinitas están representadas por restos de crinoideos desarticulados con una orientación aleatoria. Esto corresponde a las características de un flujo de detritos, donde la acumulación de sedimentos fue baja. El alto grado de desarticulación se interpreta que los restos estuvieron el tiempo suficiente sobre el fondo marino, el cual permitió la desintegración del tejido blando y posteriormente un extenso retrabajo.

\section{CONCLUSIONES}

Se reconoció un conjunto de crinoideos pensilvánicos en sedimentos siliciclásticos en afloramientos de la formación La Joya en Sierra Agua Verde, México. Este conjunto corresponde a una encrinita formada por quince especies parataxonómicas de crinoideos.

Desde el punto de vista tafonómico, la encrinita corresponde a una concentración de tipo sedimentológico formada por restos alóctonos con un alto grado de desarticulación y empaquetamiento.

Las características tafonómicas dominantes en los ejemplares estudiados fueron en primer lugar la desarticulación y en segundo la corrasión y reorientación, esto como resultado de un transporte en un flujo de densidad.

La característica de bioincrustación presentó un grado muy bajo y la colonización ocurrió después de la muerte de los crinoideos, lo cual indica un tiempo de resiliencia intermedio en la interfase agua-sedimento.

Los resultados tafonómicos permiten concluir que las encrinitas formadas durante el Pensilvánico (Atokano) en la formación La Joya, Sonora, corresponden a depósitos de una rampa externa dominada por tormentas en un ambiente mixto siliciclástico carbonatado.

\section{AGRADECIMIENTOS}

La investigación se llevó a cabo en el marco de los Proyectos CONACyT No. 165826; ECOS Francia-CONACYT México No. M13U01 "Evolución de los Ecosistemas del Paleozoico de México" y CONACyT No. 235970 "La Revolución del Cámbrico y la gran radiación del Ordovícico en el norte de México y Oaxaca”. Se agradece a Greta Ramírez Guerrero la revisión y corrección del texto.

\section{RESUMEN}

Los crinoideos poseen un extenso registro fósil y fueron un componente mayor de las comunidades marinas del Paleozoico. La encrinita es una acumulación común decrinoideos donde los especímenes están totalmente desarticulados del Ordovícico al Jurásico. En Sonora, México, el esqueleto de crinoideos fue alterado durante la diagénesis y remplazado por sílice. La corrosión fue alta y la incrustación baja, hay evidencia de disolución y compactación. Este es un tipo de acumulación sedimentológica en un ambiente de alta energía de organismos alóctonos transportados en un flujo de escombros, depositado por debajo de la base de olas de tormentas en una rampa externa. Los resultados pueden ser correlacionables con encrinita regional prevalente en ciclotemas del Pensilvánico de América del Norte.

Palabras clave: Encrinita regional; procesos tafonómicos; concentraciones sedimentológicas; Pensilvánico; Carbonífero. 


\section{REFERENCIAS}

Ausich, W.I. (1997). Regional encrinites: a vanished lithofacies. In Brett, C.E., Baird, G.C., (Eds.), Paleontological Events: Stratigraphic, Ecologic \& Evolutionary Implications (pp. 509-519). Columbia University Press: New York.

Ausich, W.I. (1980). A model for niche differentiation in Lower Mississippian crinoid communities. Journal of Paleontology, 54, 273-288.

Ausich, W.I., \& Baumiller, T.K. (1993). Taphonomic method for determining muscular articulations in fossil crinoids. Palaios, 8, 477-484.

Ausich W. I., \& Sevastopulo G. D. (1994). Taphonomy of Lower Carboniferous crinoids from the Hook Head Formation, Ireland. Lethaia, 27, 245-256.

Ausich, W.I., Brett, C E., \& Hess, H. (1999). Taphonomy. In Hess, H., Ausich, W.I., Brett, C.E., Simms, M.J. (Eds.), Fossil crinoids (pp. 50-59). Cambridge University Press: USA.

Baumiller, T.K., \& Ausich W.I. (1992). The broken-stick model as a null hypothesis for crinoid stalk taphonomy and as a guide to the distribution of connective tissue in fossils. Paleobiology, 11, 105-119.

Baumiller, T.K., \& Gahn, F.J. (2004). Testing predatordriven evolution with Paleozoic crinoid arm regeneration. Science, 305, 1453-1455.

Baumiller, T.K., \& Hagdorn, H. (1995). Taphonomy as a guide to functional morphology of Holocrinus, the first post-Paleozoic crinoid. Lethaia, 28, 221-228.

Baumiller, T.K., Llewellyn G., Messing C.G., \& Ausich W.I. (1995). Taphonomy of isocrinid stalks; influence of decay \& autotomy. Palaios, 10, 87-95.

Brachert, T.T., Betzler, C., Braga, J.C., \& Martin, J.M (1998). Microtaphofacies of a warm-temperate carbonate ramp (Uppermost Tortonian/Lowermost Messinian, Southern Spain). Palaios, 13, 459-475.

Boolootian, R.A. (1966). Physiology of Echinodermata. Interscience Publisher. New York.

Brett, C.E., \& Bordeaux, Y.L. (1990). Taphonomy of brachiopods from a Middle Devonian shell bed; implications for the genesis of skeletal accumulations. In MacKinnon, D.L., Lee, D.E., Campbell, J.D. (Eds.), Brachiopods through time. (pp. 219-226). Rotterdam: A.A. Balkema.

Burchette, T.P., \& Wright, V.P. (1992). Carbonate ramp depositional systems. Sedimentary Geology, 79, 3-57.

Carozzi, A.V., \& Soderman, G.W. (1962). Petrography of Mississippian (Borden) Crinoidal limestone at Stobo, Indiana. Journal of Sedimentary Petrology, $32,397-414$
Clayer, F. (2012). Sediment dynamics and stratigraphic architecture of a lower Silurian storm-dominated carbonate ramp, Anticosti Island, Québec, Cana$d a$. Unpublished Master in Science thesis. OttawaCarleton Geoscience Center \& University of Ottawa. Ottawa, Canada.

Cornell, S.R., Brett, C.E., \& Sumrall C.D. (2003). Paleoecology \& Taphonomy of an Edrioasteroid-Dominated Hardground Association from Tentaculitid Limestones in the Early Devonian of New York: A Paleozoic Rocky Peritidal Community. Palaios, 18, 212-224.

Donovan, S.K. (1996). Testing the Marine \& Continental Fossil Records: Comment. Geology, 24, 381-381.

Donovan, S.K. \& Clements, D. (2002). Taphonomy of large echinoids; Meoma ventricosa (Lamarck) from the Pliocene of South Carolina. Southeastern Geology, 41, 169-176.

Donovan, S.K., \& Gordon, C.M. (1993). Echinoid taphonomy and the fossil record: supporting evidence from the Plio-Pleistocene of the Caribbean. Palaios, $8,304-306$

Donovan, S.K., \& Pickerill, R.K. (1995). Crinoid columns preserved in life position in the Silurian Arisaig Group of Nova Scotia, Canada. Palaios, 10, 362-370.

Donovan, S.K., \& Schmith, D.A. (2001). Survival of crinoid stems following decapitation: evidence from the Ordovician \& palaeobiological implications. Lethaia, 34, 263-270.

Dornbos, S.Q., \& Bottjer, D.J. (2001). Taphonomy and environmental distribution of helicoplacoid echinoderms. Palaios, 16, 197-204.

Flügel, E. (2004). Microfacies of Carbonate Rocks, Analysis, Interpretation and Application. Berlin: Springer.

Gahn, F.J., \& Baumiller T.K. (2004). A Bootstrap Analysis for Comparative Taphonomy Applied to Early Mississippian (Kinderhookian) Crinoids from the Wassonville Cycle of Iowa. Palaios, 19, 17-38.

Gilinsky, N.L., \& Bennington, J.B. (1994). Estimating numbers of whole individuals from collections of body parts: a taphonomic limitation of the paleontological record. Paleobiology, 20, 245-258.

Ginsburg, R.N. (2005). Disobedient sediments can feedback on their transportation, deposition \& geomorphology. Sedimentary Geology, 175, 9-18.

Gluchowski, E. (2005). Epibionts on Upper Eifelian crinoid columnals from the Holy Cross Mountains, Poland. Acta Palaeontologica Polonica, 50, 315-328.

Goebel, K.A. (1991). Interpretation of the Lower Mississippian Johana Limestone \& the implications for the 
Antler orogenic system. Unpublished Ph.D. dissertation, University of Arizona, Tucson.

Gordon C. M., \& Donovan S. K. (1992). Disarticulated echinoid ossicles in paleoecology and taphonomy: the last interglacial Falmouth Formation of Jamaica. Palaios, 7, 157-166.

Greenstein B. J. (1991). An integrated study of echinoid taphonomy: predictions for the fossil record of four echinoid families. Palaios, 6, 519-540.

Holterhoff, P. F. (1996). Crinoid biofacies in Upper Carboniferous cyclothems, midcontinent North America: faunal tracking and the role of regional processes in biofacies recurrence. Palaeogeography, Palaeoclimatology, Palaeoecology, 127, 47-81.

Hunter A.W., \& Clark N.D.L. (2009). The palaeoecology of two Scottish encrinites: Jurassic crinoid assemblages from the Trotternish Peninsula, Isle of Skye, Scotland. Scottish Journal of Geology, 45, 169-176.

Hunter A.W., \& Underwood C.J. (2009). Palaeoenvironmental control on distribution of crinoids in the Bathonian (Middle Jurassic) of England and France. Acta Palaeontologica Polonica, 54, 77-98.

Hunter, A.W., \& Zonneveld, J.P. (2008). Palaeoecology of Jurassic encrinites: Reconstructing crinoid communities from the Western Interior Seaway of North America. Palaeogeography, Palaeoclimatology, Palaeoecology, 263, 58-70.

Kidwell, S.M., \& Baumiller T. (1990). Experimental disintegration of regular echinoids: roles of temperature, oxygen, and decay thresholds. Paleobiology, $16,247-271$.

Kidwell, S.M., \& Jablonski, D. (1983). Taphonomic feedback: ecological consequences of shell accumulations. In Tevesz, M.J.S. \& McCall, P.L. (Eds.), Biotic Interactions in Recent and Fossil Benthic Communities. (pp. 195-248). Topics in Geobiology 3, Springer.

Kidwell, S.M., Fürsich, T.F., \& Aigner, T. (1986). Conceptual framework for the analysis and classification of fossil concentrations, Palaios, 1, 228-238.

Kroh, A., \& Nebelsick J.H. (2003). Echinoid assemblages as a tool for palaeoenvironmental reconstruction-an example from the Early Miocene of Egypt. Palaeogeography, Palaeoclimatology, Palaeoecology, 201, 157-177.

Kroh, A., \& Nebelsick J.H. (2010). Echinoderms and Oligo-Miocene carbonate systems: potential applications in sedimentology and environmental reconstruction. International Association of Sedimentology, Special Publication 42, 201-228.

Llewellyn, G., \& Messing, C.G. (1993). Compositional and taphonomic variations in modern crinoid-rich sediments from the deep-water margin of a carbonate Bank. Palaios, 8, 554-573.

Meyer, D.L., \& Meyer, K.B. (1986). Biostratinomy of recent crinoids (Echinodermata) at Lizard Island, Great Barrier Reef, Australia. Palaios, 1, 294-302.

Meyer, D.L., \& Milsom, C.V. (2001). Microbial sealing in the biostratinomy of Uintacrinus lagerstätten in the Upper Cretaceous of Kansas and Colorado, USA. Palaios, 16, 535-546.

Meyer, D.L., Ausich, W.I. \& Terry, R.E. (1989). Comparative taphonomy of echinoderms in carbonate facies: Fort Payne Formation (Lower Mississippian) of Kentucky and Tennessee. Palaios, 4, 533-552.

Moore, R.C., \& Jeffords, R.M. (1968). Classification and nomenclature of fossil crinoids based on studies of dissociated parts of their columns. The University of Kansas Paleontological Contributions, 46: 1-14.

Nebelsick J. (1999a). Taphonomic comparison between Recent and fossil sand dollars. Palaeogeography, Palaeoclimatology, Palaeoecology, 149, 349-358.

Nebelsick, J.H. (1999b). Taphonomy of Clypeaster fragments; preservation and taphofacies. Lethaia, $32,241-252$.

Nebelsick, J.H. (2004). Taphonomy of Echinoderms: introduction and outlook, en Heinzeller, T., Nebelsick, J.H. (eds.), Echinoderms. München, Taylor \& Francis Group, 471-477.

Nebelsick, J.H. \& Kroh, A. (2002). The stormy path from life to death assemblages: the formation and preservation of mass accumulations of fossil sand dollars. Palaios, 17, 378-393.

Nelson, C.S. (1988). An introductory perspective on nontropical shelf carbonates. Sedimentary Geology, 60, 3-12.

Ochoa-Granillo, A., \& Sosa-León, P. (1993). Geología y estratigrafía de la Sierra Agua Verde, con énfasis en el Paleozoico. Unpublished thesis, Universidad de Sonora, Sonora, México.

Oji, T., \& Amemiya, S. (1998). Survival of crinoid stalk fragments and its taphonomic implications. Paleontological Research, 2, 67-70.

Parsons, K.M., Brett, C.E. \& Miller, K.B. (1988). Taphonomy \& depositional dynamics of Devonian shellrich mudstone. Palaeogeography, Palaeoclimatology, Palaeoecology, 63, 109-139.

Perry, C. (1996). The rapid response of reef sediments to change in community composition: implications for time averaging and sediments accumulation. Journal of Sedimentary Research, 66, 459-467. 
Phelps, W.T., \& Droser, M.L. (2003). Depositional processes and facies of the Early Mississippian Joana Limestone: An example of encrinite formation within a foreland basin setting, Western United States. American Association of Petroleum Geology Annual Convention.

Schneider, J.A. (1989). An anomalous specimen of the unstalked Cretaceous crinoid Uintacrinus socialis Grinnell, 1876: regeneration or taphonomy? Journal of Paleontology, 63, 897-900.

Schwarzacher, W. (1963). Orientation of crinoids by current action. Journal of Sedimentary Petrology, $33,580-586$.

Sepkoski, J.J., Jr. (1981). A factor analytic description of the Phanerozoic marine fossil record. Paleobiology, 7, 36-53.

Sumrall, C.D. (2001) Paleoecology and taphonomy of two new edrioasteroids from a Mississippian hardground in Kentucky. Journal of Paleontology, 75, 136-146.
Thomka, J.R., Mosher, D., Lewis, R.D. \& Pabian, R.K. (2012). The utility of isolated crinoid ossicles and fragmentary remains in taphonomic and paleonvironmental analysis: an example from the Upper Pennsylvanian of Oklahoma, United States. Palaios, 27, 465-480.

Thomka, J.R., Lewis, R.D., Mosher, D., Holterhoff, P.F., León y León, I.A. \& Pabian, R.K. (2011). Microstratigraphy and comparative taphonomic analysis of the upper core shale of a Pennylvanian cyclothem: Keys to the recognition of subtle cyclic deposition. Gulf Coast Assocation of Geological Societies Transactions, 61, 463-481.

Wetzel, A. \& Meyer C.A. (2006). The Dangers of HighRise Living on a Muddy Seafloor: An Example of Crinoids from Shallow-Water Mudstones (Aalenian, Northern Switzerland). Palaios, 21, 155-167.

Wilson, J.L. (1975) Carbonate facies in geologic history, New York, Springer- Verlag: 471. 